\title{
Design of Micro-Bluetooth Motion Acquisition System
}

\author{
Benkun Bao, ${ }^{1,2}$ Senhao Zhang, ${ }^{1,2}$ Mostafa Orban, ${ }^{1,2,3}$ Donghai Qiu, ${ }^{1}$ Hongbo Yang, ${ }^{1,2}$ \\ and Xiankai Cheng $\mathbb{1}^{1}$ \\ ${ }^{1}$ Suzhou Institute of Biomedical Engineering and Technology, Chinese Academy of Sciences, Suzhou 215163, China \\ ${ }^{2}$ School of Biomedical Engineering, University of Science and Technology of China, Hefei 230026, China \\ ${ }^{3}$ School of Mechanical Engineering Shoubra Faculty of Engineering, Banha University, Qaliobia 13518, Egypt
}

Correspondence should be addressed to Xiankai Cheng; chengxk@sibet.ac.cn

Received 5 March 2021; Revised 7 April 2021; Accepted 25 April 2021; Published 13 May 2021

Academic Editor: Yiwei Wang

Copyright ( 2021 Benkun Bao et al. This is an open access article distributed under the Creative Commons Attribution License, which permits unrestricted use, distribution, and reproduction in any medium, provided the original work is properly cited.

To realize the digitization of infant motion information and explore more abundant human health information with motion information to facilitate early treatment, a micro-Bluetooth motion acquisition system is designed. The low-power design of the micro-Bluetooth motion capture sensor in the system and intelligent algorithm for optimizing the precision of the infant movement measured angles can realize the system's sustainable use and data reliability. With the help of collecting and analyzing human arm, leg, and head movement information, we can recognize that the system can carry out more research and experiments on natural infant movements.

\section{Introduction}

Studies have found that the movements of infants and young children who do not express themselves have a strong connection with their physical health, such as cerebral palsy $[1,2]$ and autism spectrum disorders $[3,4]$. The infant's nervous system development has the advantage of the rapid stage with remarkable plasticity and excavation that enables us to discover some diseases by analyzing the health of babies through the infant movement. The early discovery of the diseases will be highly significant for the problematic babies' rehabilitation process [5]. In the meantime, using the movement of the infants is less complex than the usual method of the large-scale brain test.

Nowadays, there are different methods for neonatal behavior assessment based on infant movement, such as neonatal behavioral, neurological assessment (NABA) [6], neonatal behavioral observation (NBO) [7], and general movements' assessment (GMs) [1, 8]. Among these standards, GMs have become an essential tool for clinical use to predict the neurodevelopmental outcome of high-risk infants, especially motion development. There are different methods to capture infant' movement by using cameras
[9-11], wearable sensors [12], and magnet tracking systems [13]. Meinecke et al. [12] introduced a sample to use and a low-cost system based on accelerometers. However, the sensor is a wired sensor, which is inconvenient and can only detect the motion of four position points. Andraž Rihar et al. [14] used a modular device called CareToy for the assessment and rehabilitation of preterm infants. This device included pressure mattresses, inertial and magnetic measurement units, and sensorized toys. A variety of assessments and predictions can be made in terms of movement assessment, but it is also more complex overall.

Each method has its advantage and disadvantage. For example, cameras are more sensitive and reliable [1] than traditional neurological examination and imaging examination. At the same time, it only captures the movement in the $\mathrm{X}$ and $\mathrm{Y}$-direction, and it cannot capture the action in the $\mathrm{Z}$ direction, and it will lose the motion's depth information. Also, it is sensitive to clothing, lighting, and occlusion. Therefore, there is a high probability that there will be some errors in the results of the motion examination. Wearable sensors and magnet tracking systems have high accuracy. But, it has a large size, is wired, is uncomfortable to wear, and affects the 
infant's movement. It can be seen from this that they have many shortcomings which cannot be ignored.

This paper will introduce a miniature wireless action acquisition system. This is the first time that Bluetooth networking technology with one master connected to six slaves has been combined with baby motion capture. Compared to other infant motion tests, this system has the advantage of being simple, convenient, light enough and not be limited by the environment or the professional, offering the possibility of expanding screening for infant-related diseases. Improving motion-captured accuracy has been done by designing a new intelligent algorithm to detect movement angles by using a gyroscope sensor. We reduced the system complexity by creating a new Bluetooth module to connect the six new slave sensors instead of using the old method of using one Bluetooth module one sensor. Our new designed sensor can be used for detecting the movement of autistic children [15] as in their infancy. They have a lot of actions to explore, such as asymmetric postures in the supine position [16], persistent asymmetric tension neck reflex, and abnormal rolling, asymmetric sitting posture, or asymmetric gait [17]. It can also be used to detect disturbed movements of newborns to screen infants with cerebral palsy [1].

\section{System Design}

2.1. System Bluetooth Design. The system includes six selfdesigned miniature Bluetooth motion capture sensors, a data summary collector, and self-designed software for analysis, processing, and display the data. The six miniature sensors collect the corresponding actions of the six human body parts (arm, legs, head, etc.). Then, these sensors convert them into digital signals, transmit them to the collector via Bluetooth, and send the data to the analysis software through the serial port to systematically analyze the collected motion data, as shown in Figure 1(a). The main chip for wireless transmission and the realization of one master-six slave is NRF52832, and the chip used for action acquisition and conversion is MPU9250. The chip used to protect the sensor and the lithium batteries during the charging and discharging process is BQ24040, as shown in Figure 1(b).

2.2. Low-Power Mode. This sensor also provides an intermittent mode and reduces the sensor's power consumption in the sleep mode as much as possible, ensuring that the average current is in a low state. Compared with the normal sleep mode, the designed sleep mode's power consumption is reduced by $99.9 \%$. The design block diagram of the sensor entering low-power sleep mode is shown in Figure 2.

The NRF52832 uses an internal DC/DC regulator to achieve lower current consumption. At the same time, an external LC filter (cut-off frequency is $50 \mathrm{~Hz}$ ) is designed to filter out the output ripple voltage to ensure voltage stability. The power consumption of mpu9250 is $3.5 \mathrm{ma}$, which accounts for more than $50 \%$ of the sensor. The low-power mode of mpu9250 needs to be designed. Otherwise, it will always be in a high-power state. So it is necessary to develop a low-power consumption mode of the chip, or the sensor as a whole will not enter the low-power consumption mode due to mpu9250 interference. Before the NRF52832 chip enters deep sleep, reset all the registers in the mpu9250, close the three-axis acquisition port of the accelerometer and the three-axis acquisition port of the gyroscope, and then set the mpu9250 enter sleep mode. At this time, it is necessary to turn off all peripherals of NRF52832 to prevent failure to enter sleep and reset the input and output ports used to avoid additional current consumption caused by high and low levels. The low-power consumption implementation steps are shown in Figure 3 shows the sensor's current consumption in different low-power consumption modes and different working states. Simultaneously, due to a large amount of calculation of the motion data solution and a large number of floating-point operations, it takes up a lot of power consumption. To reduce the power consumption of the sensor, we calculate the data in the sensor, and part of the algorithm is moved to the software. Finally, the detailed current values of the broadcast time and the connection transmission data time under the same test conditions in each case are shown in Figure 3. The abscissa of this picture adds low-power steps step by step from left to right. This figure shows that the entire sensor cannot enter the lowpower stage before mpu9250 is specifically designed for lowpower consumption. Only after mpu9250 is turned off, the low-power design of NRF528322 will officially play a role.

2.3. Angle Algorithm Design. The raw data collected by the mpu9250 motion chip is noisy, and the obtained motion information fluctuates wildly, so an algorithm for processing the raw data has been designed. In the offset correction of acceleration and angular velocity, the design of extracting, averaging, subtracting offset, and quadratic averaging ensures that the sensor can still obtain a zero offset value as long as it is at rest and does not need to maintain a specific position. It can increase the flexibility of calibration and reduce the position required for calibration.

It also used an ellipsoid fitting correction algorithm for the magnetic field data of each sensor. By acquiring the raw magnetic field data of the sensor in 8 directions, the initial origin of the magnetic field is roughly located by the maximum and minimum interpolation method, and then, the least square method is cyclically performed 8000 times to locate the best magnetic field origin. We can get the corrected magnetic field data by subtracting the best magnetic field origin value from the original magnetic field value. It can increase the flexibility of correction and ensure the authenticity of the raw data, and the data of the sensor angle at the extreme position can be changed synchronously. It can be seen in Figure 4(b) that when the magnetic field is not calibrated, the angle value of the three axes when the sensor rotates $270^{\circ}$ horizontally back and forth has a high delay. In particular, yaw cannot truly show the angle of rotation. As for the angle in Figure 4(c), it is a three-axis angle curve diagram of the sensor at the same starting position as Figure 4(b) after the magnetic field is corrected. The angles of the three axes can truly show the angle of rotation. Therefore, it can be known that 


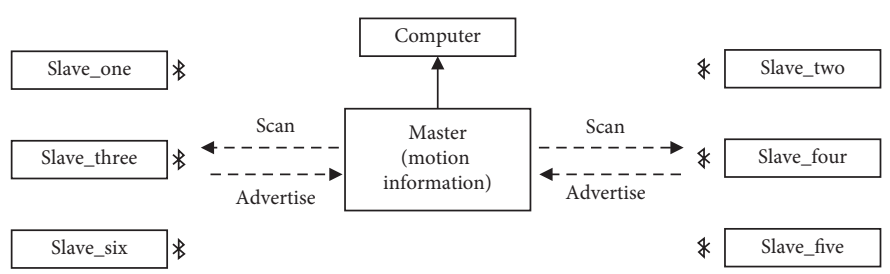

(a)

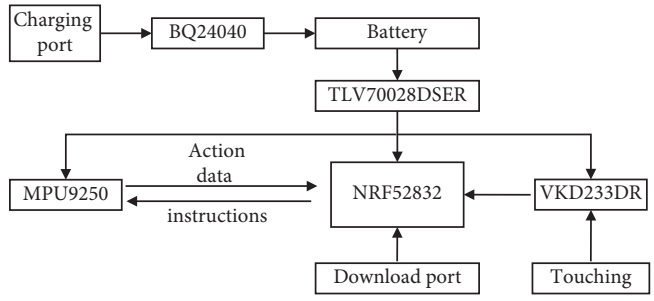

(b)

Figure 1: (a) Overall system structure and (b) sensor circuit block diagram.

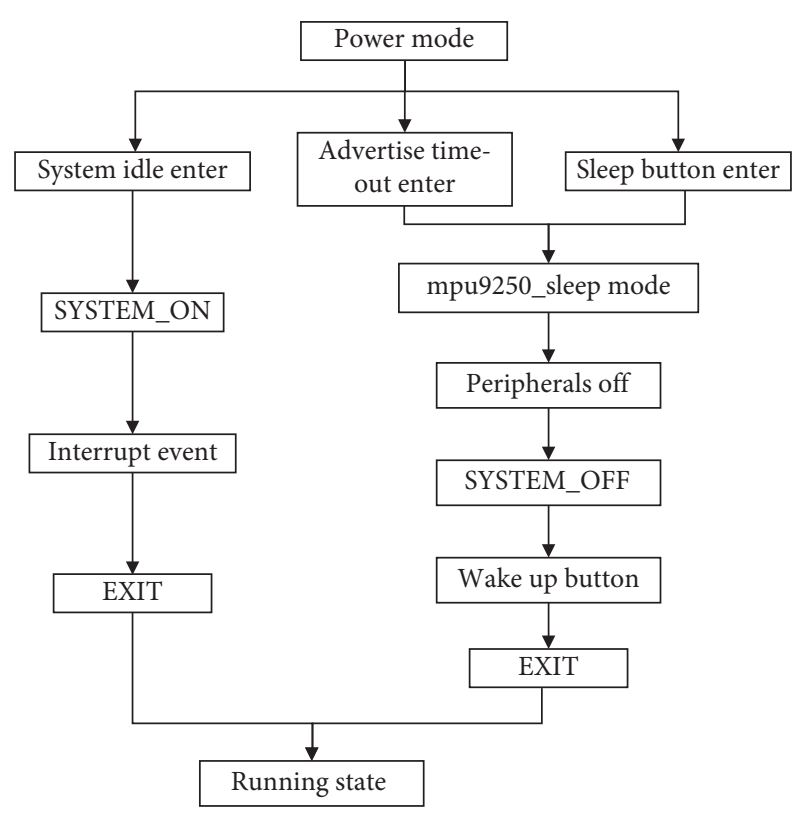

Figure 2: Low power mode design.

when the magnetic field is not corrected, its yaw value cannot obtain the actual rotation angle. A sliding filter with a depth of 4 is applied to the data in order to ensure the smoothness of the data and a certain degree of data time followability. For the final acquisition of the angle, an adaptive complementary filtering Mahony algorithm is designed and applied so that the algorithm for solving the angle can change the relevant parameters ( $\mathrm{Kp}, \mathrm{Ki}$ ) according to the actual motion situation. It will improve the dynamic performance of the obtained angle value. The angle value obtained by the corrected magnetic field is fused by secondary algorithm fusion so that the authenticity of the angle value can be guaranteed. The drift of the pitch, yaw, and roll three-axis angle values obtained by this algorithm is solved. Within 250 seconds, the drift range of the minimum pitch axis is within $0.9^{\circ}$, and the maximum drift is within $2^{\circ}$ on the yaw axis. The angle of the roll axis is within $1.5^{\circ}$, as shown in Figure 4(a).

\section{Human Experiment Verification}

The Bluetooth wireless motion acquisition sensor system had been built, as shown in Figure 5. The board size of the Bluetooth Motion Capture sensor is $11 * 16.38 * 0.8 \mathrm{~mm}$, as shown in Table 1, and the size of the rechargeable lithium battery used is $10 * 12 * 3 \mathrm{~mm}$, which is the smallest volume of similar products on the market. In Figure 5, the computer software system can process the motion information data of various parts of the body obtained from the sensor and display the analyzed information in the computer interface

At the same time, the overall naked weight of a single sensor is $1.6 \mathrm{~g}$, with a shell weight of $2.9 \mathrm{~g}$. The light quality guarantees that it fits on the human skin and will not affect the movement due to the weight. Use nonstimulating silica gel (model: 2475P) to attach the six sensors to the two arms of the human body near the wrists, the legs near the outside of the wrists, the center of the forehead of the head, and the upper part of the navel point with a finger width. The specific placement positions of the six sensors on the human body are shown in Figure 6(a).

The main purpose of this system experiment was to simulate high-frequency movements of infants with an adult tester. The effectiveness of the system in capturing the characteristics of typical movements $[18,19]$ was then evaluated. With the help of the designed sensors, the corresponding data information was obtained through symmetrical movements of the head, arms, and legs and variable acceleration [19], respectively. As shown in Figure 6(a), the motion of the test arm is divided into normal rocking and abnormal rocking. The test of legs movement is the simultaneous stretching movement of both legs shown in Figure 6(b) and the head swings from the right side to the left side shown in Figure 6(c). The experiment verifies the performance of the system and analyzes the motion characteristics by collecting the data information of these movements. The system for the data analysis is working as flow, and the microsensors transmit raw motion data to the data collection collector at a frequency of $30 \mathrm{~Hz}$. The motion data is processed and analyzed by the software on the computer, and the image is displayed in real time on the computer screen; the original data and the processed data are also saved to the database for secondary deep data processing. Each packet of the sensor data has a total of 24 bytes, which is a custom data format, including the data header ( 4 bytes) and the sensor name code (1 byte). After the six sensors are connected to the data acquisition, it takes $10 \mathrm{~s}$ for the sensor initialization and calibration to be completed. The movement of infants and young children is mostly a simultaneous movement of both arms and legs; therefore, when designing the exercise type, the simultaneous exercise of both hands and legs is 


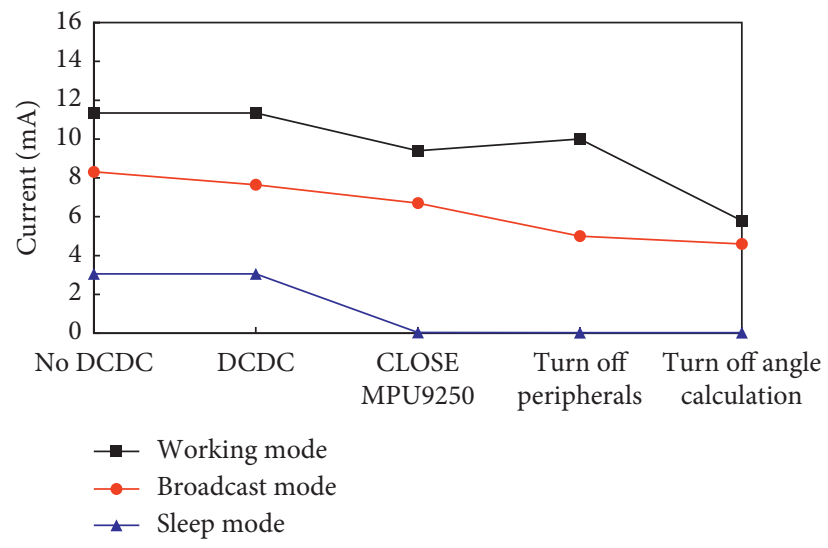

Figure 3: Current consumption under different conditions.

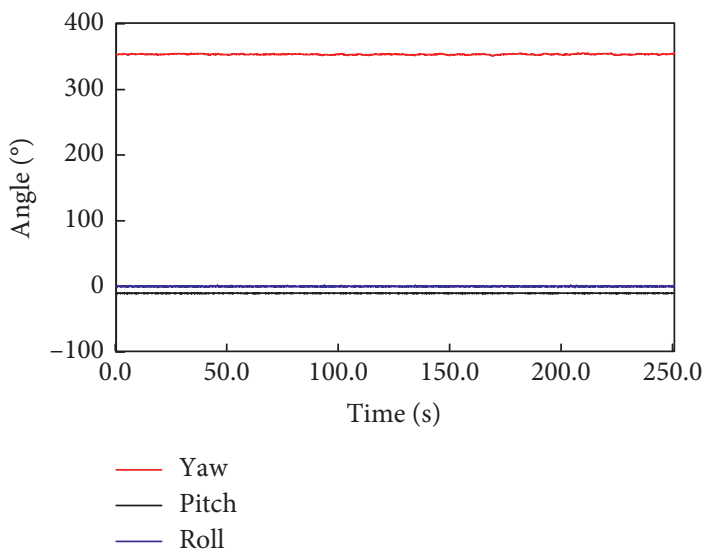

(a)

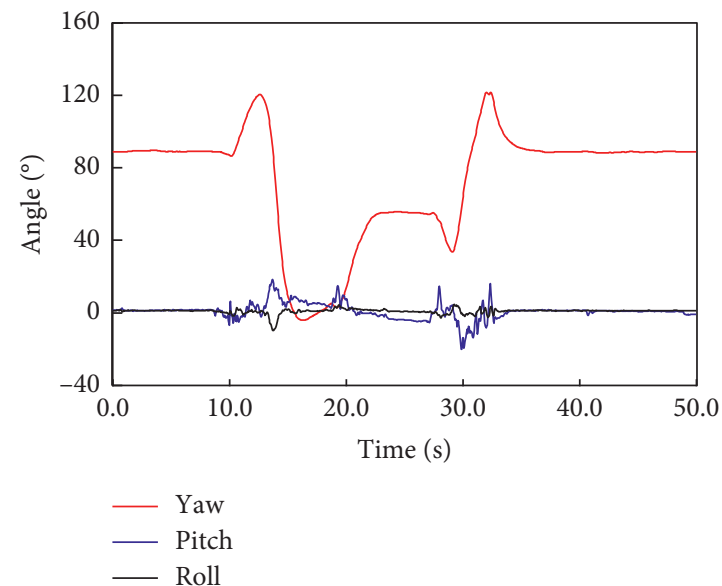

(b)

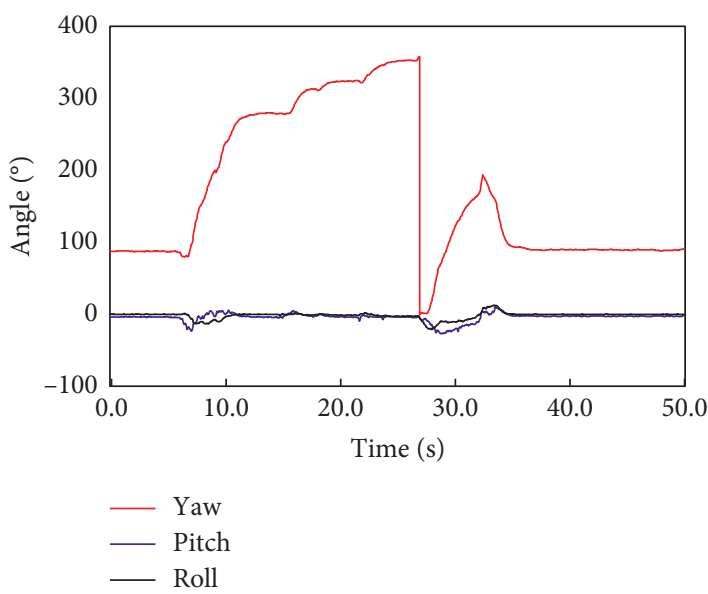

(c)

Figure 4: (a) The sensor is resting is the calculated angle value. (b) Angle change before magnetic field correction. (c) Angle change after magnetic field correction.

mainly considered, while the single of one arm or leg exercise is used as an auxiliary.

The angle and acceleration values obtained under the normal shaking of the arm are shown in Figures 7(a), 7(c), and $7(\mathrm{~d})$, passing and abnormal arm shaking are shown in
Figures 7(b), 7(e), and 7(f). Comparing the acceleration with normal shaking, the acceleration generated by abnormal shaking has more peaks. The angle value of abnormal shaking has more burrs than normal shaking, which indicates that the movement has obvious unsmoothness. As for 


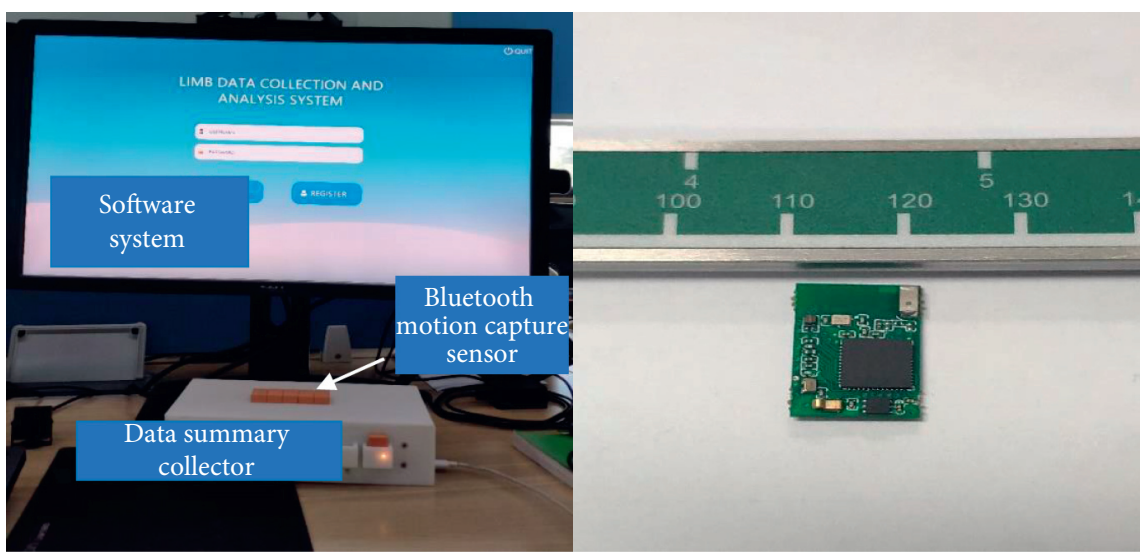

Figure 5: Overall system and sensor circuit board diagram.

TABLE 1: Net size comparison of sensors with the same function.

\begin{tabular}{lcccc}
\hline Sensor & My sensor & LCM20948 & BM160 & JY61 \\
\hline Size & $11 * 16.38$ & $15.32 * 26.54$ & $22 * 22$ & $15.24 * 15.24$ \\
\hline
\end{tabular}

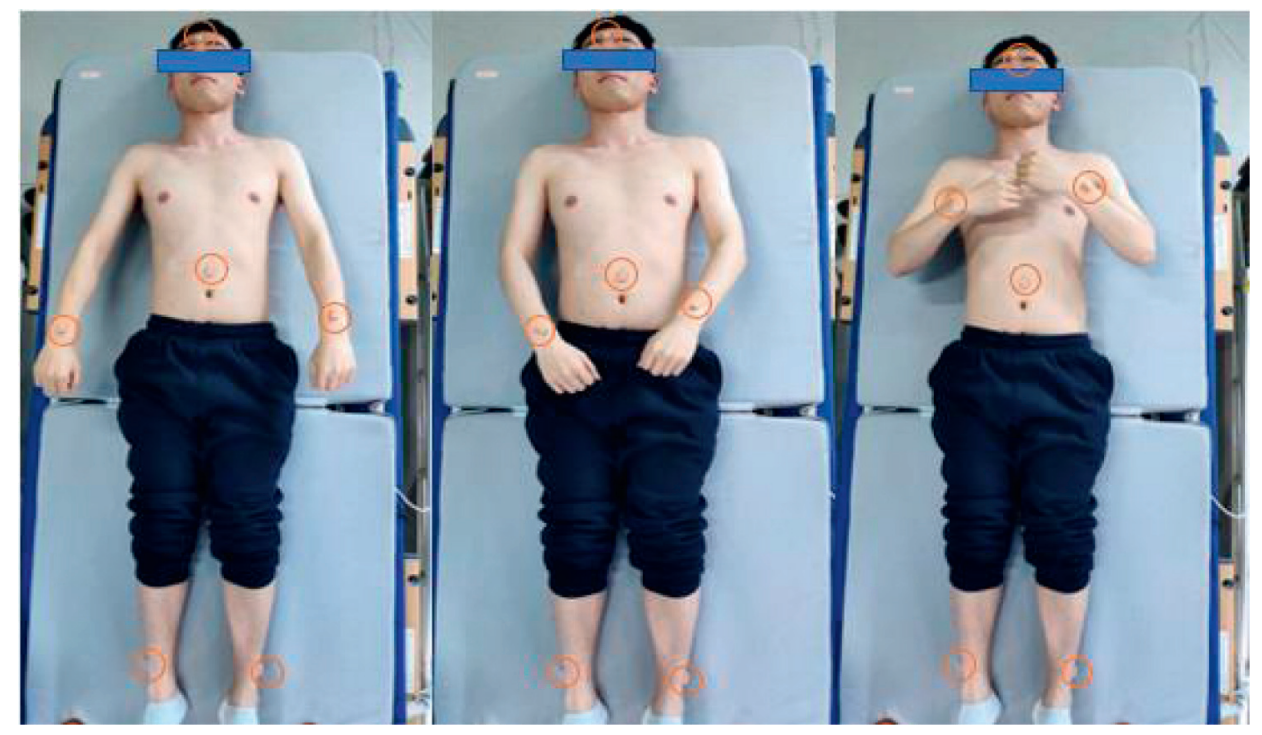

(a)

FIgURE 6: Continued. 


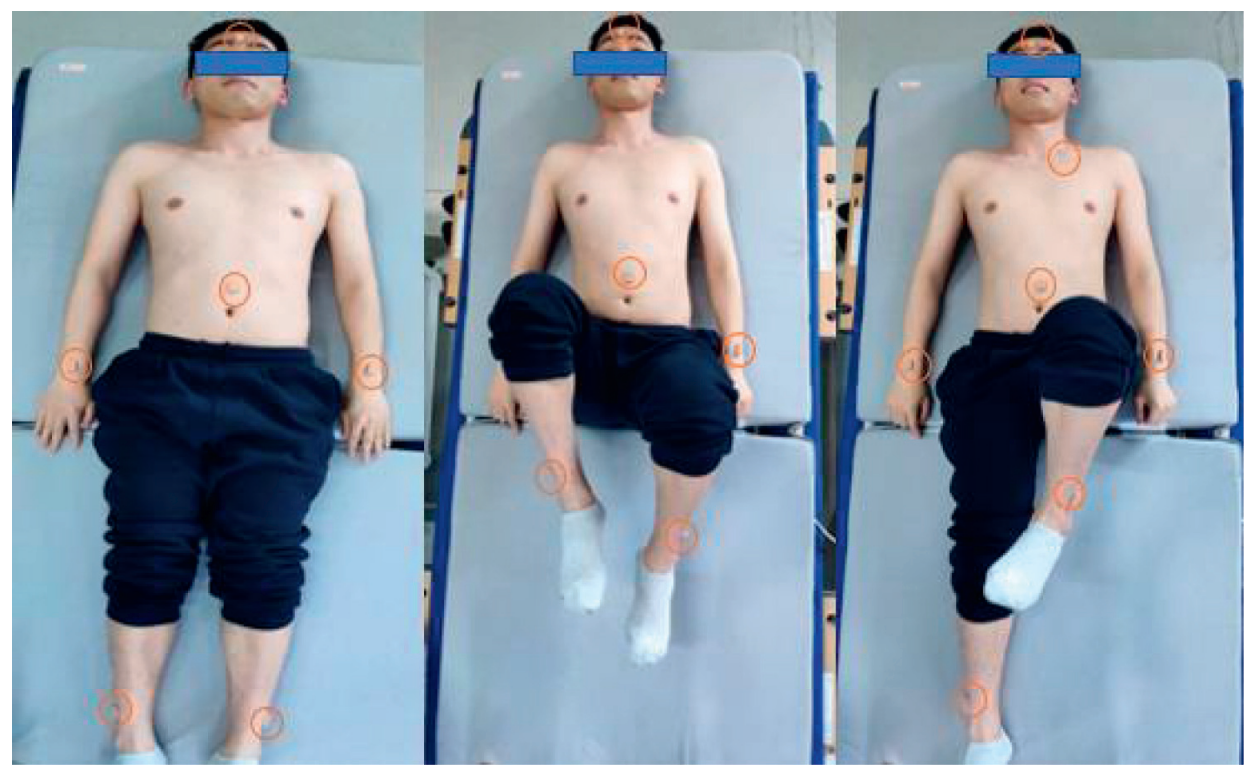

(b)

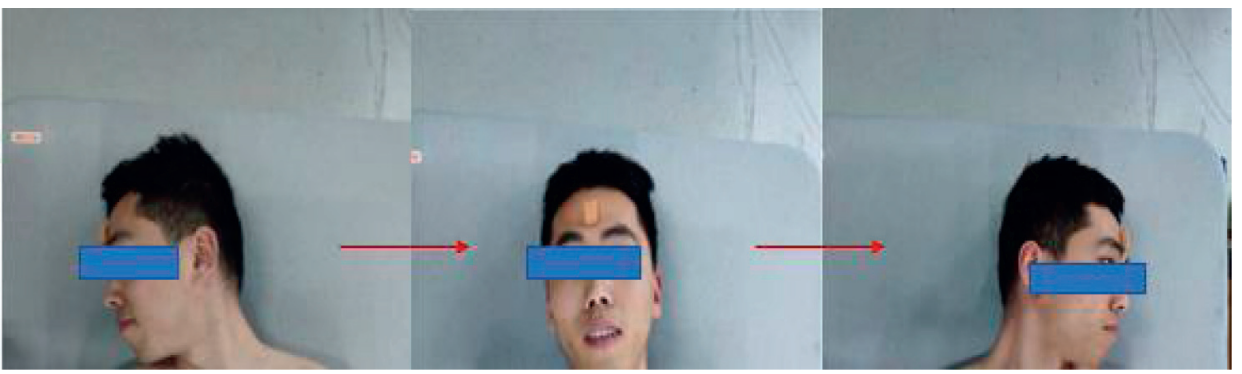

(c)

Figure 6: The demonstration test involves slight shaking of the hands, legs, and head. (a) The tester's arm movement diagram. (b) The tester's leg movement diagram. (c) The tester's head-shaking picture from side to side.

the angle in Figure 7(h), this angular curve represents the arm moving at normal speed five times. It can be seen fast motion of the arm angle curve changes in Figure 7. In yaw, pitch, and roll, the sensor can quickly capture the corresponding five peaks in both fast and normal speed motion, and the peaks are free of excessive spurious waves.

The leg movements of the test subjects are shown specifically in Figure 6(b), and the curve of leg movement represents five times of double-legged movements and five times of single-legged movements, respectively. As shown in Figure $8(\mathrm{a})$, the acceleration data of the five times leg movements were displayed, and the acceleration curves in all three directions produced significant pole effects, as well as data fluctuations, at the curve positions corresponding to the movements. In the angular data for the five times leg movements shown in Figure 8(c), similar data characteristics are even more evident. The value of yaw in this case even appears as a rectangular wave-like waveform. In the change of acceleration curve for one leg lift in Figure 8(b), there are two data fluctuations with opposite polarity when the tester's leg moves in the air as well as when it lands on the bed. The angular curve change of one leg lift action in Figure $8(d)$, where the yaw value is clearly characterized, and the data analysis can be performed based on the yaw value for data truncation analysis. The presence of data features for the entire leg lift action verifies the feasibility of the later data analysis.

During the infant test, the infant's head moves at a high frequency as it lies flat on the bed, so a unique rotation sequence is designed, which is middle-left-middle-right-middle-leftmiddle-right, and this movement lasts for 50 seconds. Every turning action captured by the miniature sensor is highly characteristic. Also, it is solid for detecting the acceleration and angle following the head rotation, as shown in Figure 9. The head is turned with complete movement from middle-leftmiddle-right as shown in Figure 9(a). Meanwhile, the acceleration value is detected by the sensor on the forehead. The miniature sensor detected the angle value on the forehead when the head turned a whole movement from middle-left-middleright as shown in Figure 9(b). For a clear view of the change of acceleration of each movement, the movement of turn the head from the center to the right is shown in Figure 9(c). In contrast, the movement of turn the head from the center to the left is shown in Figure 9(d). It can be seen in Figure 7 that the typical motion of the head has a clear signal signature. The acceleration and angular line plots of standard head motion resemble 

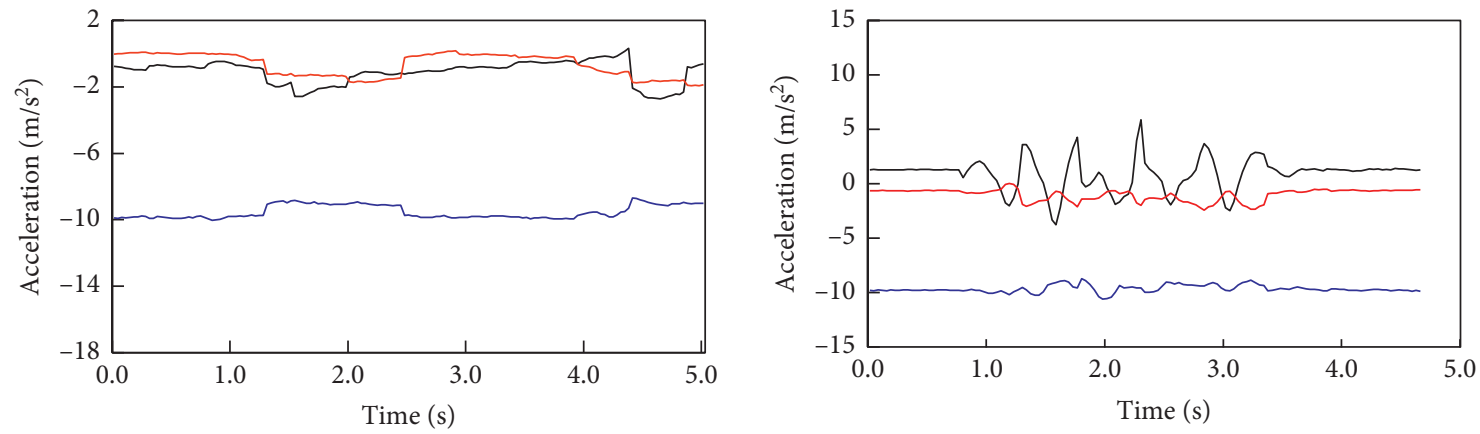

$-\mathrm{Ax}$
$-\mathrm{Ay}$
$-\mathrm{Az}$

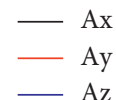

(a)

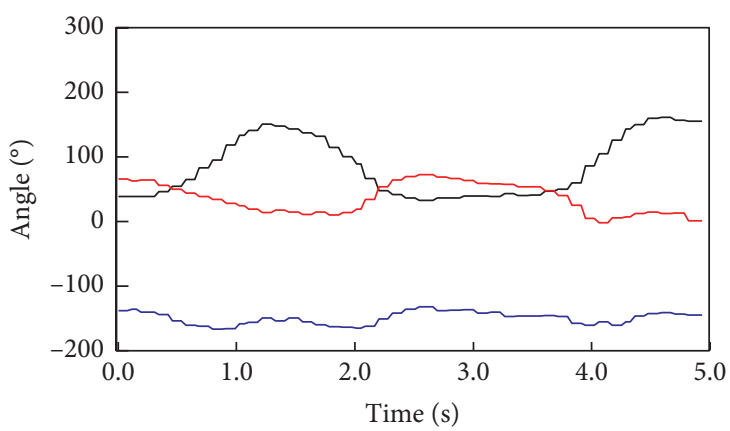

(b)

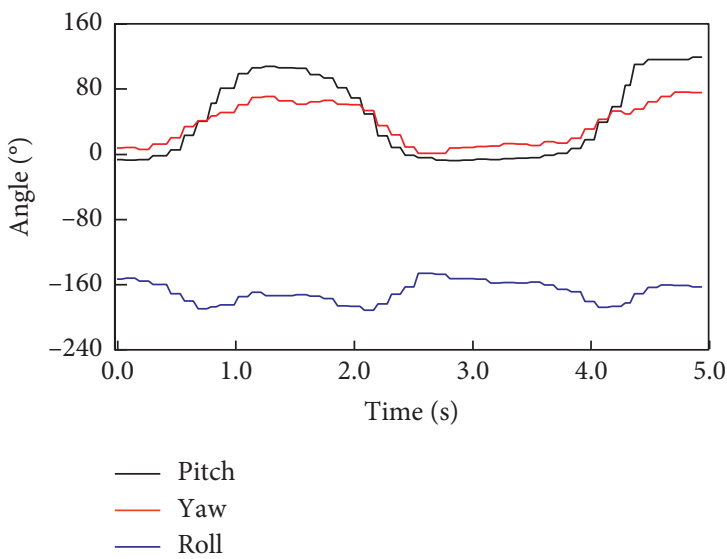

(c)
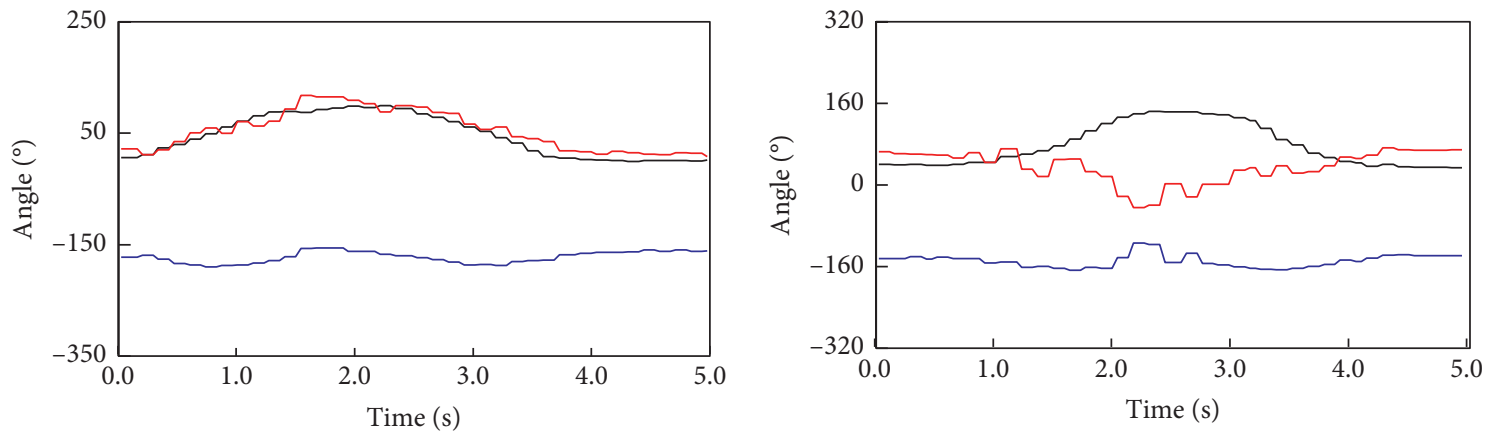

Pitch
- Yaw
Roll

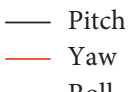

(e)

(f)

FIGURE 7: Continued. 


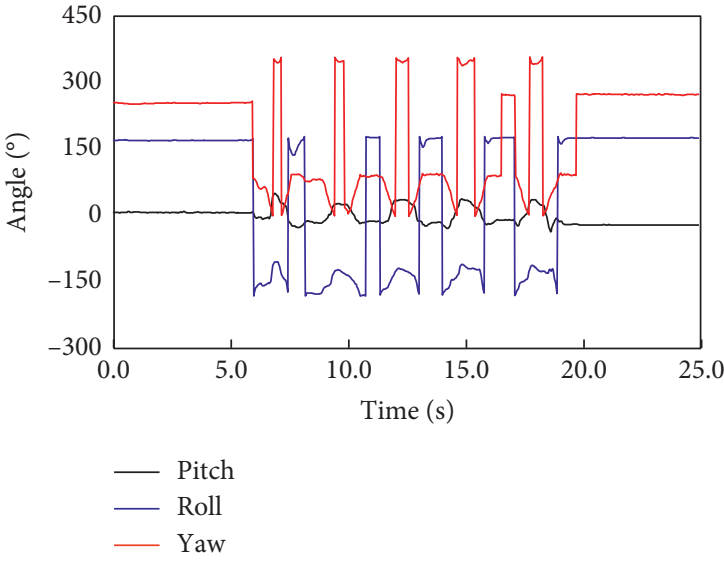

(g)

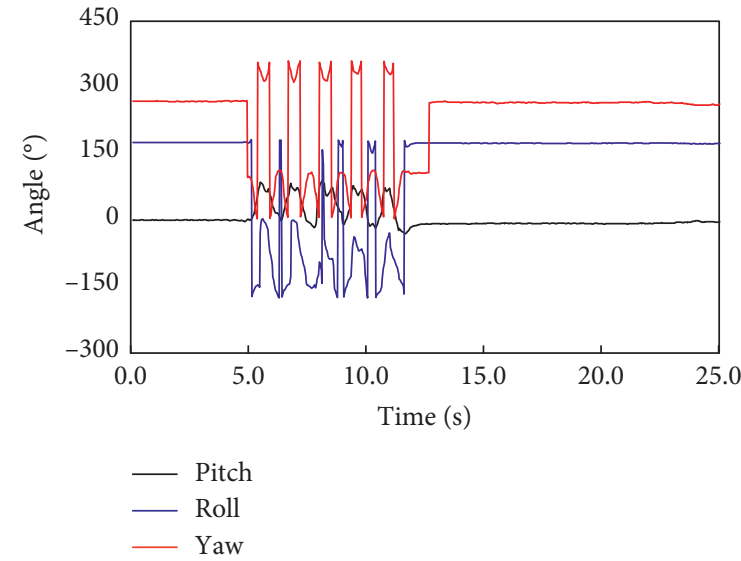

(h)

Figure 7: (a) The acceleration value obtained by the normal movement of the left arm, as shown in Figure 6(a). (b) The acceleration value obtained by the left arm in the unstable state of the arm shaking, as shown in Figure 6(a). (c) The angle value of the normal movement of the left arm. (d) The angle value of the normal movement of the right arm. (e) The angle value obtained under the unstable state of the left arm shaking. (f) The angle value obtained under the unstable state of the right arm shaking. (g) Change in angle for five normal arm movements. (h) Change in angle for five normal arm movements.
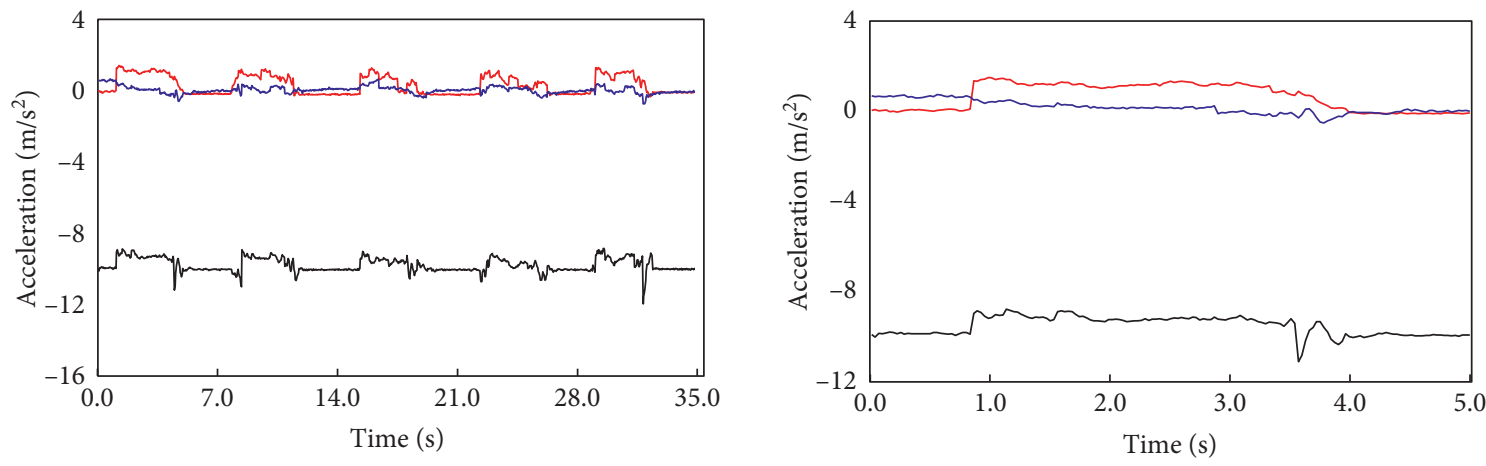

$-\mathrm{Ax}$
$-\mathrm{Ay}$
$-\mathrm{Az}$

- Ax

— Ay

$-\mathrm{Az}$

(a)
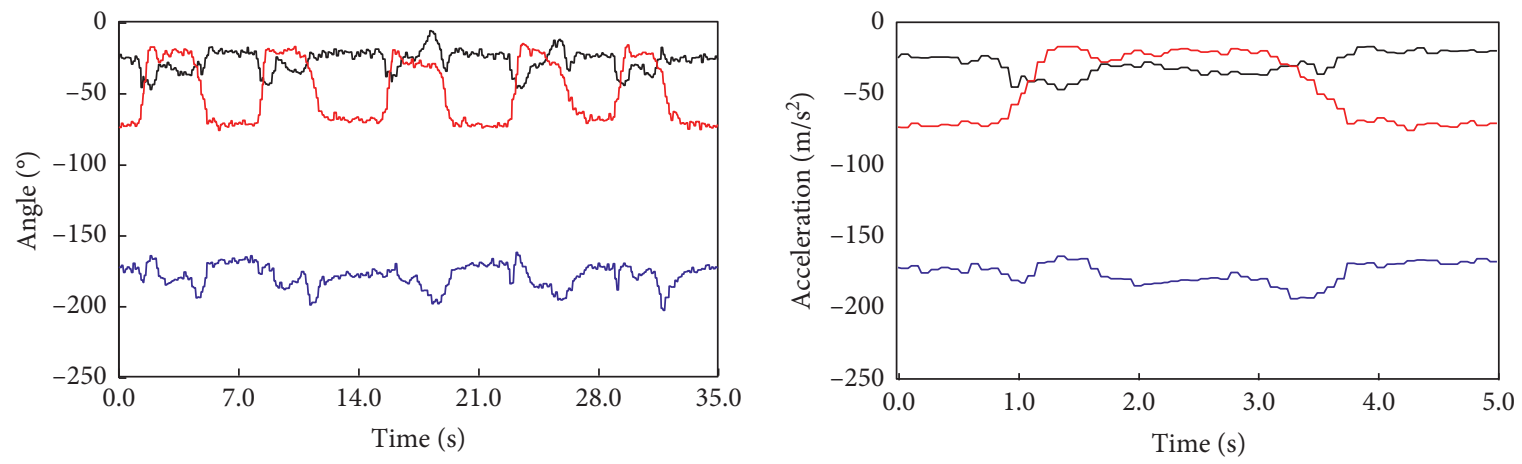

— Pitch
— Yaw
— Roll

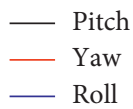

(c)

(d)

Figure 8: (a) Acceleration change curve of five leg lifts. (b) Acceleration change curve of single-leg lift. (c) Angle change curve of five leg lifts. (d) Angle change curve of single-leg lift. 


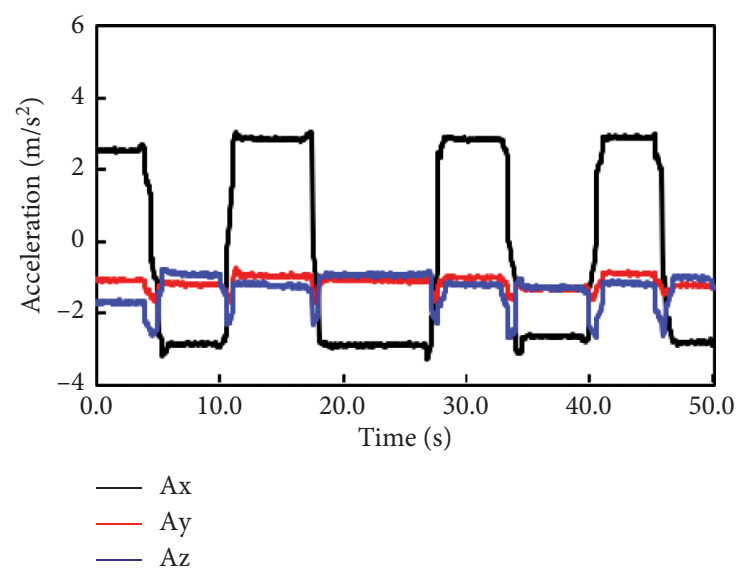

(a)

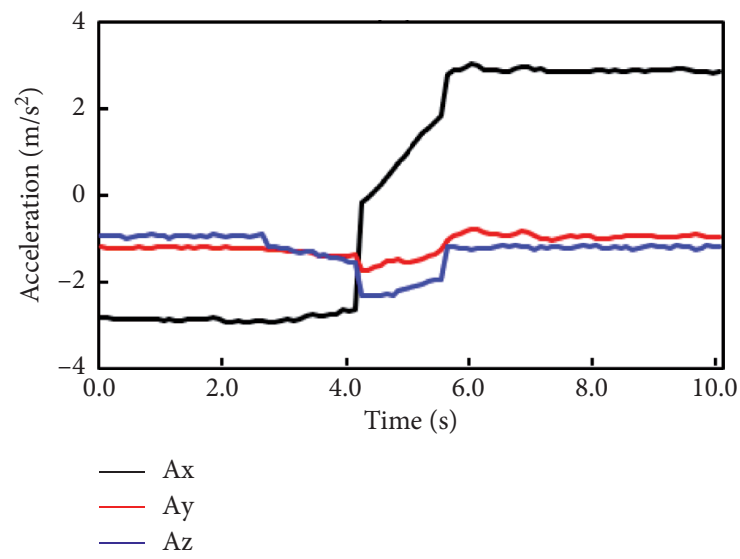

(c)

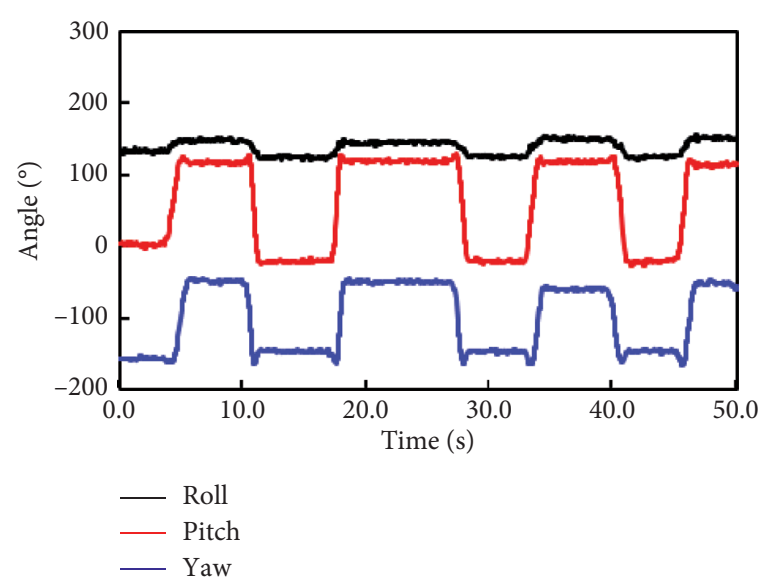

(b)

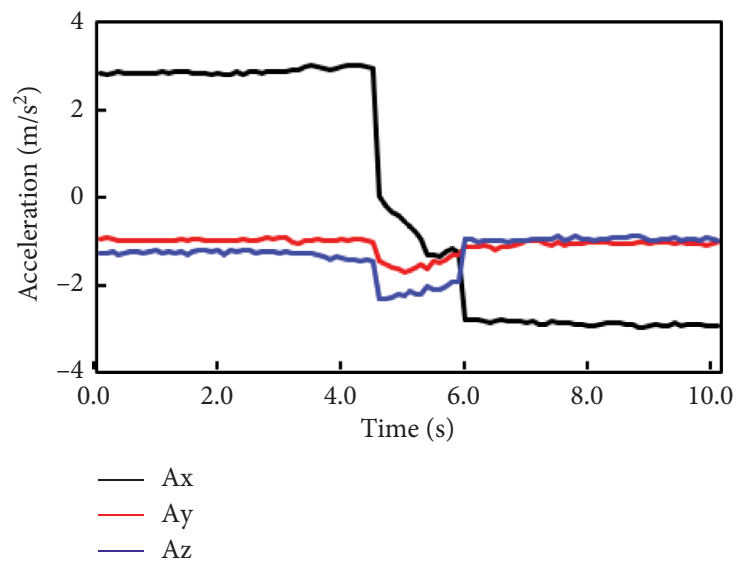

(d)

Figure 9: Movement data when the head is turned left and right. (a) Head motion acceleration diagram. (b) Head motion angle diagram. (c) Head left rotation acceleration diagram. (d) Head right rotation acceleration diagram.

rectangular pulse plots, so the head motion's quality can be easily discriminated.

\section{Conclusions}

A miniature Bluetooth wireless sensor and acquisition system has been designed and verified to be feasible in adult simulation experiments which is used to detect the movement of infants and young children. The main advantages of the system are as follows. (1) The designed sensor is light in weight and small in size, which produce less interference to the movement. (2) The sensor used one master-six slave Bluetooth technology, which significantly reduces the complexity of the system. (3) The system can realize and capture any slight difference of the infant motion, which is a great help for the medical field to check the infant health condition; at the current situation, the theoretical and technical verification of the system applied on adult's body confirmed the applicability of our system for collecting the movement preciously. After this test, the system runs stably, and it can realize the external analysis of the motion data, which is a good start for further research.

\section{Data Availability}

The data used to support the findings of this study are available from the corresponding author upon request.

\section{Conflicts of Interest}

The authors declare that they have no conflicts of interest.

\section{References}

[1] H. F. R. Prechtl, C. Einspieler, G. Cioni, A. F. Bos, F. Ferrari, and D. Sontheimer, "An early marker for neurological deficits after perinatal brain lesions," The Lancet, vol. 349, no. 9062, 1997.

[2] N. Kanemaru, H. Watanabe, H. Kihara et al., "Specific characteristics of spontaneous movements in preterm infants at term age are associated with developmental delays at age 3 years," Developmental Medicine \& Child Neurology, vol. 55, no. 8, pp. 713-721, 2013.

[3] A. N. Bhat, R. J. Landa, and J. C. Galloway, "Current perspectives on motor functioning in infants, children, and adults 
with autism spectrum disorders," Physical Therapy, vol. 91, no. 7, p. 1116, 2011.

[4] P. Teitelbaum, O. Teitelbaum, J. Nye, J. Fryman, and G. Ralph, "Movement analysis in infancy may be useful for early diagnosis of autism," Proceedings of the National Academy of Sciences of the United States of America, vol. 95, no. 23, 1998.

[5] C. M. Bann, J. L. Wallander, B. Do et al., "Home-based early intervention and the influence of family resources on cognitive development," Pediatrics, vol. 137, no. 4, 2016.

[6] V. M. Moore, M. J. Davies, K. J. Willson et al., "Dietary composition of pregnant women is related to size of the baby at Birth 1,2," Journal of Nutrition, vol. 134, no. 7, pp. 1820-1826, 2004.

[7] J. K. Nugent, J. D. Bartlett, A. Von Ende, and C. Valim, "The effects of the newborn behavioral observations (NBO) system on sensitivity in mother-infant interactions," Infants \& Young Children, vol. 30, no. 4, pp. 257-268, 2017.

[8] L. Dimitrijevi, B. Bjelakovi, C. Hristina et al., "Assessment of general movements and heart rate variability in prediction of neurodevelopmental outcome in preterm infants," Early Human Development, vol. 99, pp. 7-12, 2016.

[9] E. Christa and F. R. Prechtl Heinz, "Prechtl's assessment of general movements: a diagnostic tool for the functional assessment of the young nervous system," Mental Retardation and Developmental Disabilities Research Reviews, vol. 11, no. 1, pp. 61-67, 2005.

[10] H. F. R. Prechtl, "General movement assessment as a method of developmental neurology: new paradigms and their consequences," Developmental Medicine \& Child Neurology, vol. 43, no. 12, pp. 836-842, 2007.

[11] N. Kanemaru, H. Watanabe, H. Kihara et al., "Jerky spontaneous movements at term age in preterm infants who later developed cerebral palsy," Early Human Development, vol. 90, no. 8, 2014.

[12] L. Meinecke, N. Breitbach-Faller, C. Bartz, R. Damen, G. Rau, and C. Disselhorst-Klug, "Movement analysis in the early detection of newborns at risk for developing spasticity due to infantile cerebral palsy," Human Movement Science, vol. 25, no. 2, 2006.

[13] H. Philippi, D. Karch, K.-S. Kang et al., "Computer-based analysis of general movements reveals stereotypies predicting cerebral palsy," Developmental Medicine \& Child Neurology, vol. 56, no. 10, 2014.

[14] A. Rihar, M. Mihelj, J. Pai et al., "Infant posture and movement analysis using a sensor-supported gym with toys," Medical \& Biological Engineering \& Computing, vol. 57, no. 197, pp. 1-13, 2018.

[15] H. Gima, H. Kihara, H. Watanabe et al., "Early motor signs of autism spectrum disorder in spontaneous position and movement of the head," Experimental Brain Research, vol. 236, no. 4, pp. 1139-1148, 2018.

[16] A. Hossein and P. Memari, "Postural control impairments in individuals with autism spectrum disorder: a critical review of current literature," Asian Journal of Sports Medicine, vol. 5, no. 3, p. e22963, 2014

[17] Z. Michele, E. Christa, D. Katrin et al., "What do home videos tell us about early motor and socio-communicative behaviors in children with autistic features during the second year of life-an exploratory study," Early Human Development, vol. 91, no. 10, pp. 569-575, 2015.

[18] T. Tsunenobu, L. G. Robert, H. Jinron et al., "Cord serum ferritin concentrations and mental and psychomotor development of children at five years of age," The Journal of Pediatrics, vol. 140, no. 2, pp. 165-170, 2002.
[19] G. C. Oa, V. Vigneron, B. Dorizzi et al., "Predicting postoperative gait in cerebral palsy," Gait \& Posture, vol. 52, no. Complete, pp. 45-51, 2017. 